\title{
Bulletin of the Seismological Society of America
}

This copy is for distribution only by

the authors of the article and their institutions in accordance with the Open Access Policy of the Seismological Society of America.

For more information see the publications section of the SSA website at www.seismosoc.org

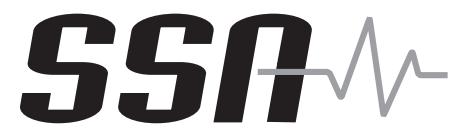

The Seismological Society of America 400 Evelyn Ave., Suite 201

Albany, CA 94706-1375

(510) 525-5474; FAX (510) 525-7204

www.seismosoc.org 


\title{
Empirical Equations for the Prediction of the Significant, Bracketed, and Uniform Duration of Earthquake Ground Motion
}

\author{
by Julian J. Bommer, Peter J. Stafford, and John E. Alarcón
}

\begin{abstract}
The complete characterization of earthquake ground motion includes the length of the interval of strong shaking as well as the amplitude and frequency content of the time series. There are relatively few published equations available for the prediction of strong-motion duration from earthquakes, which may in part be a consequence of the fact that the duration of shaking has generally not been considered in structural engineering. Recognizing that there are many applications for which an estimate of the duration of ground motion is needed, this study presents new empirical predictive equations for a number of definitions of strong-motion duration using the records from the Next Generation of Attenuation (NGA) global database of accelerograms from shallow crustal earthquakes. The equations can be used to estimate ground-motion durations from shallow crustal earthquakes of magnitude between $M_{\mathrm{w}} 4.8$ and 7.9 at distances up to $100 \mathrm{~km}$ from the source.
\end{abstract}

\section{Introduction}

Earthquake ground motion can be characterized by many different parameters, each of which reflects some particular feature of the shaking such as the peak amplitudes, the frequency content, or the energy carried in the signal. A complete characterization of the ground motion must include a measure of its duration, or more specifically the duration of that part of the signal that is considered to be strong. A large number of definitions of strong-motion duration have been put forward in the literature, although these can generally be grouped into three generic categories (bracketed, uniform, and significant) and then classified by whether the amplitude or energy thresholds used for their measurement are absolute or relative to the peak value in the recording (Bommer and Martínez-Pereira, 1999).

There is no clear consensus as to which of the multiple definitions of duration is to be preferred, which probably reflects the fact that different definitions may be more or less suitable for different applications. The real issue is that by itself the duration of the motion conveys very little about the ground shaking and its potential for causing damage, and its influence generally needs to be quantified in relation to another parameter of the ground motion. This is clear in most methods for the assessment of liquefaction hazard, where the capacity of the ground motion to trigger loss of shear strength is a function of both the amplitude of the motion and its duration or the number of cycles. The apparent role of duration in the response of the ground or structures will depend on the primary ground-motion parameter used to characterize the shaking. For two accelerograms with similar amplitudes, the record with longer duration will generally be more damaging, whereas for two records with the same energy content, it is likely that the record with shorter duration would cause more destruction (Bommer and MartínezPereira, 1999). For this reason, prediction of duration by itself may be of limited value, and the long-term objective of this study is to develop vector predictions of duration in conjunction with other ground-motion parameters related to amplitude and energy content (Bazzurro and Cornell, 2002). The derivation of equations for the stand-alone prediction of duration measures is primarily a contribution toward this goal and serves to explore the behavior of this parameter, but the equations presented herein may also be of use for a number of applications.

The importance of ground-motion duration in geotechnical engineering is universally recognized, the parameter most widely adopted for this purpose usually being the number of cycles of motion (Liu et al., 2001; Green and Terri, 2005; Hancock and Bommer, 2005). Surprisingly, the number of cycles is found to be very poorly correlated with duration (Bommer et al., 2006). Therefore, in order to address the need for direct prediction of the numbers of cycles, Stafford and Bommer (2009) have recently derived empirical equations for this purpose.

The relevance of duration to structural response, however, is a subject of considerable debate. Hancock and Bommer (2006) reviewed a large number of studies on this subject and demonstrated that conclusions regarding the influence of duration on structural damage depend on several factors including the type of structure examined, the other parameters used to characterize the ground motion, and the structural parameters used to quantify damage. Those studies that characterize damage by maximum response 
parameters find little or no influence of duration, whereas those using cumulative measures, such as hysteretic energy loss, find that the duration has an appreciable influence on the level of damage. For structures whose stiffness and strength degrade under the action of seismic shaking, such as masonry buildings, the influence of duration can be pronounced (e.g., Bommer et al., 2004), whereas the response of steel structures, for example, would be expected to be far less sensitive to duration. In their study of masonry structures, Bommer et al. (2004) used the elastic acceleration response as the primary parameter to characterize the ground motions and noted that because this parameter has some degree of correlation with the duration of the motion, the influence of the latter may be partially concealed. In order to investigate this issue, Hancock and Bommer (2007) used spectral matching to produce a suite of accelerograms with almost identical elastic response ordinates but with very different durations, which were then applied to the analysis of a multidegree-of-freedom structure. The results confirmed that if cumulative parameters are used to measure the damage, then the duration of the motion is found to have a marked influence on the inelastic structural response. However, current approaches to earthquake-resistant design and structural analysis generally take no account of the duration of the motion, and this parameter is not explicitly included in the provisions of any seismic design code known to the authors. One possible exception to this was the introduction of the dominant earthquake magnitude-provided as the basis for selecting the response period at which the constant displacement plateau begins-in the 2003 National Earthquake Hazards Reduction Program (NEHRP) provisions (BSSC, 2004), from which duration could also be inferred. The general absence of direct reference to duration in seismic design codes may partly explain why relatively little attention has been given to the prediction of duration and why so few equations have been published for the prediction of duration compared to more widely used parameters such as response spectral ordinates (e.g., Douglas, 2003).

One current development in earthquake engineering that may provide additional motivation to derive equations for the prediction of duration is displacement-based seismic design (e.g., Priestley et al., 2007). Some approaches to this design methodology use an equivalent linear system to represent the inelastic deformation of a structure, with extended response periods to model the decrease in stiffness as damage progresses and increased equivalent viscous damping to represent the dissipation of energy through hysteresis. The spectral ordinates for damping levels higher than the nominal $5 \%$ of critical usually assumed in seismic design codes are generally obtained by applying simple scaling factors-which are functions of the target damping level-to the 5\%-damped displacement ordinates. In exploring the wide variation among such scaling factors in design codes and in the literature, Bommer and Mendis (2005) found that the ratios of spectral ordinates at different damping levels are strongly dependent on the duration of the ground motion. Stafford,
Mendis, and Bommer (2008) have subsequently derived new scaling factors that are simultaneously functions of the target damping level and the duration of the ground motion.

Both to address the emerging needs in structural engineering and as a first step in the development of vector predictions of ground-motion parameters coupled with duration, this study uses a large global database of earthquake accelerograms to derive equations for the prediction of a number of measures of strong-motion duration. In a way, this article is an update and extension of the study of Kempton and Stewart (2006), which presented equations for the prediction of significant duration derived from the Next Generation of Attenuation (NGA) dataset; this article explores alternative functional forms for such equations and additionally presents equations for the prediction of other definitions of duration.

\section{Strong-Motion Database}

The database employed for this study is essentially the same as that used by Stafford and Bommer (2009) to derive equations for the prediction of the number of cycles of motion. The records are extracted from the database compiled for the NGA project (Chiou et al., 2008). The selection follows similar reasoning to that of Abrahamson and Silva (2008) to include as many records as possible from shallow crustal earthquakes worldwide but limiting the distance range of records from non-western U.S. events to $100 \mathrm{~km}$ to prevent differences in regional crustal structure from becoming significant. While Abrahamson and Silva (2008) retained records from western U.S. events with distances exceeding $100 \mathrm{~km}$, in this study we remove these records. An additional constraint applied by Stafford and Bommer (2009) was to exclude records from events with depths to the top of the rupture greater than $15 \mathrm{~km}$. The associated metadata for the final dataset of 2406 records were taken directly from the NGA flatfile. The records are from 114 earthquakes with moment magnitudes in the range from 4.8 to 7.9 and with the following distribution of styles-of-faulting: 56 strike slip, 35 reverse or reverse oblique, and 23 normal or normal oblique. The recordings are from sites with a wide range of shear-wave velocities, ranging from just over 100 up to $2000 \mathrm{~m} / \mathrm{sec}$.

The explanatory variables adopted in this study are the moment magnitude, $M_{\mathrm{w}}$, the closest distance from the fault rupture, $R_{\text {rup }}(\mathrm{km})$, depth to the top of rupture, $Z_{\text {tor }}(\mathrm{km})$, and the average shear-wave velocity over the uppermost $30 \mathrm{~m}$ at the site, $V_{S 30}(\mathrm{~m} / \mathrm{sec})$. The influence of the style-of-faulting is represented by a single parameter, $F_{\mathrm{rv}}$, which takes a value of 1 for reverse and reverse-oblique events and 0 for normal and strike-slip ruptures. As explained in the following section of the article, however, not all of these variables are included in every predictive model.

The durations were calculated from the horizontal components of the records according to a number of different definitions. The first of these is the significant duration, 
$D_{S}$, which is defined as the interval between the times at which different specified values of Arias intensity are reached. Because the thresholds of Arias intensity used in this case are relative to the total Arias intensity of the record, the symbol $D_{\mathrm{SR}}$ is used, with the second subscript indicating the relative threshold. Two definitions of $D_{\mathrm{SR}}$ are used, both starting at the point at which $5 \%$ of the total Arias intensity is reached. The first definition measures the duration up to the point at which $75 \%$ of the total is reached, while the other extends to $95 \%$ of the Arias intensity; the former definition is intended to capture the energy from the body waves whereas the latter includes the full wave train.

The next definition of duration employed is the bracketed duration, $D_{B}$, which is defined as the interval between the first and last excursion of a specified threshold acceleration. The final definition is the uniform duration, $D_{U}$, which differs from the bracketed duration in that it only considers the intervals for which the ground acceleration is above the threshold, and therefore $D_{U}$ is always shorter than $D_{B}$ for a given record. For these two definitions, absolute thresholds are used, whence the durations are represented by the symbols $D_{\mathrm{BA}}$ and $D_{\mathrm{UA}}$. Three threshold accelerations are used with each definition, these being $0.025,0.05$, and $0.10 \mathrm{~g}$, whence in total six duration definitions are employed. In using absolute thresholds of acceleration, clearly for any component record for which the peak ground acceleration (PGA) is below the threshold, the duration is zero, and hence as the thresholds increase, the number of component records with nonzero durations available for regression decreases. The number of records available for the regression analysis on each definition of duration are given in Table 1.

In calculating the durations, consideration must be given to the treatment of the two horizontal components of each accelerogram. Various different schemes have been used for parameters related to the amplitude of the motion (Beyer and Bommer, 2006), with the most popular in recent years becoming the geometric mean of the values from the two horizontal components and variations of this definition (Boore et al., 2006; Watson-Lamprey and Boore, 2007). For durations it is not clear what physical interpretation one could give to the geometric mean, and for the $D_{\mathrm{BA}}$ and $D_{\mathrm{UA}}$ definitions those records with one component having zero duration would obviously create problems. The approach adopted instead is to use both components of the records, which in effect represents the random component of motion. The geometric mean component was originally introduced in strong-motion studies as a surrogate for the random component, and it has subsequently been pointed out that although median values of the two definitions may be almost identical, the associated aleatory variability in each case will differ due to the component-to-component variability (Baker and Cornell, 2006). In this study we use the method of Boore (2005) to calculate the component-tocomponent variability and then use this to transform the standard deviation (sigma) of the random component to that for the geometric mean. For this reason, Table 1 also indicates the number of pairs of horizontal components available in each case (i.e., both having nonzero duration values) for calculating the component-to-component variability.

\section{Regression Analyses}

As noted in the Introduction, relatively few equations have been published, at least in recent years, for the prediction of ground-motion duration, so there is relatively little guidance available on suitable functional forms. However, Abrahamson and Silva (1996) have proposed a functional form for the prediction of significant duration that is based upon seismological considerations. More recently, Kempton and Stewart (2006) have implemented the same basic functional form for the scaling with respect to both magnitude and distance. The rationale behind the selection of this functional form is that the significant duration at the source can be assumed equivalent to the source duration. For the prediction of significant duration at a given site, this source duration must be modified to account for the effects of the travel path and near-surface site effects, as well, potentially, as other phenomena. From basic seismological theory, the source duration may be assumed equal to the reciprocal of the corner frequency, which may in turn be related to a measure of the earthquake size as in equation (1):

$$
D_{\mathrm{SR}}\left(R_{\mathrm{rup}}=0\right) \equiv \frac{1}{f_{c}\left(M_{0}, \Delta \sigma\right)}=\frac{1}{4.9 \times 10^{6} \beta}\left(\frac{M_{0}}{\Delta \sigma}\right)^{1 / 3} \text {. }
$$

Here, $\beta$ is the shear-wave velocity of the crust in the vicinity of the source, $\Delta \sigma$ is the stress drop, and $M_{0}$ is the seismic moment. Both Abrahamson and Silva (1996) and Kempton

Table 1

Sizes of Datasets Used for the Derivation of the Empirical Predictive Models for Different Measures of Duration

\begin{tabular}{lccccrrrr}
\hline & $D_{\mathrm{SR}}(5-75 \%)$ & $D_{\mathrm{SR}}(5-95 \%)$ & $D_{\mathrm{UA}}(0.025)$ & $D_{\mathrm{UA}}(0.050)$ & $D_{\mathrm{UA}}(0.100)$ & $D_{\mathrm{BA}}(0.025)$ & $D_{\mathrm{BA}}(0.050)$ & $D_{\mathrm{BA}}(0.100)$ \\
\hline Eqs & 114 & 114 & 112 & 104 & 95 & 112 & 104 & 95 \\
Recs & 2406 & 2406 & 1918 & 1454 & 853 & 1918 & 1456 & 854 \\
Comps & 4812 & 4812 & 3699 & 2681 & 1471 & 3706 & 2699 & 1487 \\
Pairs & 2406 & 2406 & 1795 & 1271 & 652 & 1797 & 1272 & 657 \\
\hline
\end{tabular}

Eqs, number of earthquakes contributing records; Recs, number of records contributing components; Comps, number of components used; and Pairs, number of records with two nonzero durations on both horizontal components. 
and Stewart (2006) allowed the stress drop to be modeled as a function of earthquake magnitude, although Kempton and Stewart (2006) only used this form for $D_{\mathrm{SR}}(5-95 \%)$ and retained a constant stress-drop model for $D_{\mathrm{SR}}(5-75 \%)$. Both studies also made the sound assumption that the marginal distribution of duration is lognormal. The base functional form that was consequently adopted by these authors for the magnitude scaling is therefore written as

$$
\begin{aligned}
& \ln D_{\mathrm{SR}}\left(R_{\text {rup }}=0\right) \\
& \quad=\ln \left(\frac{1}{4.9 \times 10^{6} \beta}\left\{\frac{\exp \left[b_{1}+b_{2}\left(M-M^{*}\right)\right]}{10^{1.5 M+16.05}}\right\}^{-1 / 3}\right) .
\end{aligned}
$$

Here, $b_{1}$ and $b_{2}$ are parameters to be obtained during the regression analysis, and $M^{*}$ is a reference magnitude that is simply selected by the analyst. The expression in equation (2) is arrived at after following physical arguments but is not a particularly elegant expression. From the perspective of regression analysis it is preferable to recast the expression in equation (2) in an equivalent, but simpler, form. Fortunately, it is straightforward to do so, as can be appreciated from equation (3):

$$
\begin{aligned}
& \ln D_{\mathrm{SR}}\left(R_{\text {rup }}=0\right) \\
&=\left\{\frac{1}{3}\left[16.05 \ln (10)-b_{1}+b_{2} M^{*}\right]-\ln \left(4.9 \times 10^{6} \beta\right)\right\} \\
& \quad+\left[\frac{1}{2} \ln (10)-\frac{1}{3} b_{2}\right] M .
\end{aligned}
$$

In the present study, the basic scaling of durations with respect to magnitude is therefore assumed to be linear and equation (2) may be expressed in the far simpler equivalent form as in equation (4), where $c_{0}$ and $m_{1}$ are parameters to be obtained via regression analysis:

$$
\ln D_{\mathrm{SR}}\left(R_{\text {rup }}=0\right)=c_{0}+m_{1} M \text {. }
$$

It is worth noting here that if the stress drop is modeled as a constant with respect to magnitude, then one should expect the coefficient $m_{1}$ to be approximately 1.15. Abrahamson and Silva (1996), for $D_{\mathrm{SR}}(5-75 \%)$, and Kempton and Stewart (2006), for $D_{\mathrm{SR}}(5-95 \%)$, found the value of $b_{2}$ to be 0.85 and 0.82 , respectively, which would indicate that the coefficient on the magnitude scaling could be reduced by about a quarter of a unit. While such considerations are useful, one must always keep in mind that although the functional form may be based upon physical reasoning, the final model is entirely empirical and care should be taken when inferring physical attributes from regression coefficients. This is particularly true in the case where terms including magnitude appear in other parts of the functional form.

For the present study, in addition to the basic scaling with earthquake magnitude described by equation (4), the functional forms that were considered included terms to capture scaling with respect to distance, shear-wave velocity, the depth to the top of the earthquake fault rupture, and styleof-faulting. For each of these effects several functional terms were tested. The selection of the final form was driven by the ability of the model to capture the scaling observed in the empirical data in combination with a desire to adopt as simple a functional form as possible. Standard statistical metrics were used to quantify the ability of a particular model to satisfy both of these criteria. In particular, likelihood ratio tests were conducted, and metrics, such as the Akaike information criteria, the Bayesian information criteria, and the restricted log-likelihood, were compared among the different models. Residuals of the empirical models were checked and the statistical significance of all of the coefficients, as well as the correlations among the coefficients, were inspected to ensure that the empirical models that were derived were robust.

The final functional form that was adopted for the prediction of significant duration, both $D_{\mathrm{SR}}(5-75 \%)$ and $D_{\mathrm{SR}}(5-95 \%)$, is given in equation (5):

$$
\begin{aligned}
\ln D_{\mathrm{SR}}(x)= & c_{0}+m_{1} M_{\mathrm{w}}+\left(r_{1}+r_{2} M_{\mathrm{w}}\right) \ln \sqrt{R_{\mathrm{rup}}^{2}+h_{1}^{2}} \\
& +v_{1} \ln V_{S 30}+z_{1} Z_{\mathrm{tor}} .
\end{aligned}
$$

The coefficients of equation (5) were obtained using the nlme package in R (Pinheiro et al., 2008; R Core Development Team, 2008). These coefficients are presented, along with associated $95 \%$ confidence intervals, in Table 2 . The coefficients $r_{1}, r_{2}, h_{1}, v_{1}$, and $z_{1}$ are determined by regression together with the coefficients $c_{0}$ and $m_{1}$ introduced earlier. The inclusion of the term representing the scaling with respect to the depth to the top of rupture may surprise some readers. However, the regression analysis indicates a very wellconstrained negative correlation between the logarithmic duration and this depth measure. Within the dataset that is used in this study there is a clear correlation between

Table 2

Parameters of the Regression Models for Significant Durations with Relative Thresholds Defined as $5-75 \%$ and $5-95 \%$ of Arias Intensity

\begin{tabular}{ccc}
\hline Parameter & $D_{\mathrm{SR}}(5-75 \%)$ & \multicolumn{1}{c}{$D_{\mathrm{SR}}(5-95 \%)$} \\
\hline$c_{0}$ & $-5.6298 \pm 0.9325$ & $-2.2393 \pm 0.8051$ \\
$m_{1}$ & $1.2619 \pm 0.1401$ & $0.9368 \pm 0.1223$ \\
$r_{1}$ & $2.0063 \pm 0.1845$ & $1.5686 \pm 0.1489$ \\
$r_{2}$ & $-0.252 \pm 0.027$ & $-0.1953 \pm 0.0219$ \\
$h_{1}$ & $2.3316 \pm-1.5293$ & $2.5^{*}$ \\
$v_{1}$ & $-0.29 \pm 0.0338$ & $-0.3478 \pm 0.0274$ \\
$z_{1}$ & $-0.0522 \pm 0.0223$ & $-0.0365 \pm 0.0202$ \\
$\tau$ & $0.3527 \pm 0.0577$ & $0.3252 \pm 0.0521$ \\
$\sigma$ & $0.4304 \pm 0.0116$ & $0.346 \pm 0.0107$ \\
$\sigma_{c}$ & $0.1729 \pm 0.0049$ & $0.1114 \pm 0.0031$ \\
$\sigma_{T, \mathrm{ARB}}$ & $0.5564 \pm 0.0372$ & $0.4748 \pm 0.036$ \\
$\sigma_{T, \mathrm{GM}}$ & $0.5289 \pm 0.0392$ & $0.4616 \pm 0.0371$ \\
\hline
\end{tabular}

The plus and minus values define the approximate $95 \%$ confidence interval for each parameter.

"Note that the parameter $h_{1}$ is fixed for the 5-95\% case, for which reason no confidence intervals are reported. 
magnitude and depth to the top of rupture, with larger events tending to have smaller depths; it is likely that this correlation is having some effect on the observed dependency. However, Kagawa et al. (2004) found that buried ruptures tend to have larger stress drops, and we believe that the observed scaling with respect to the depth to the top of rupture can be primarily explained as a result of this. As the depth of the event increases, the significant duration decreases for an earthquake of the same magnitude. If the energy released by an event is related to the earthquake magnitude, then an increase in ground-motion amplitudes with increasing stress drop for a given magnitude would simultaneously lead to a shorter significant duration as is predicted by our model.

The influence of the style-of-faulting was not found to be statistically significant for these durations, and inspection of the residuals with respect to this parameter confirmed that its inclusion in the predictive model is not warranted.

In Table 2 the total standard deviation of the models is discussed, the physical interpretation of the geometric mean of duration is not as clear as it is for amplitude-based measures of ground motion. The regression analyses are therefore conducted using individual components, and the two stated total standard deviations correspond to the total standard deviation obtained directly from the regression analysis, $\sigma_{T, \mathrm{ARB}}$, and that corresponding to the geometric mean duration, which is obtained through an adjustment of the $\sigma_{T, \mathrm{ARB}}$ represented in two ways, $\sigma_{T, \mathrm{ARB}}$ and $\sigma_{T, \mathrm{GM}}$. As previously

to account for the component-to-component variability, $\sigma_{c}$. The relationships among these variance components are described formally in equations (6) and (7) in which $\tau$ and $\sigma$ represent the interevent and intraevent standard deviations, respectively:

$$
\begin{gathered}
\sigma_{T, \mathrm{ARB}}=\sqrt{\tau^{2}+\sigma^{2}+\sigma_{c}^{2}}, \\
\sigma_{T, \mathrm{GM}}=\sqrt{\sigma_{T, \mathrm{ARB}}^{2}-\sigma_{c}^{2}} .
\end{gathered}
$$

In deriving the models for significant duration, a distinction was made between aftershocks and mainshocks. The standard deviations of the aftershock recordings were found to be $\sim 15 \%$ larger than the mainshock records. In Table 2 the standard deviations that are presented correspond to those for mainshock events only.

Figures 1 and 2 show the residuals of the two definitions of significant duration against the four predictor variables included in equation (5), which confirm that the functional form and fitting procedures are robust.

For the bracketed and uniform durations, the regression approach of Stafford (2008) was implemented in order to account for the presence of components with zero-valued durations. This approach requires the prediction of the probability that each component will not result in a zero-valued duration. These values are equivalent to the probability that the PGA
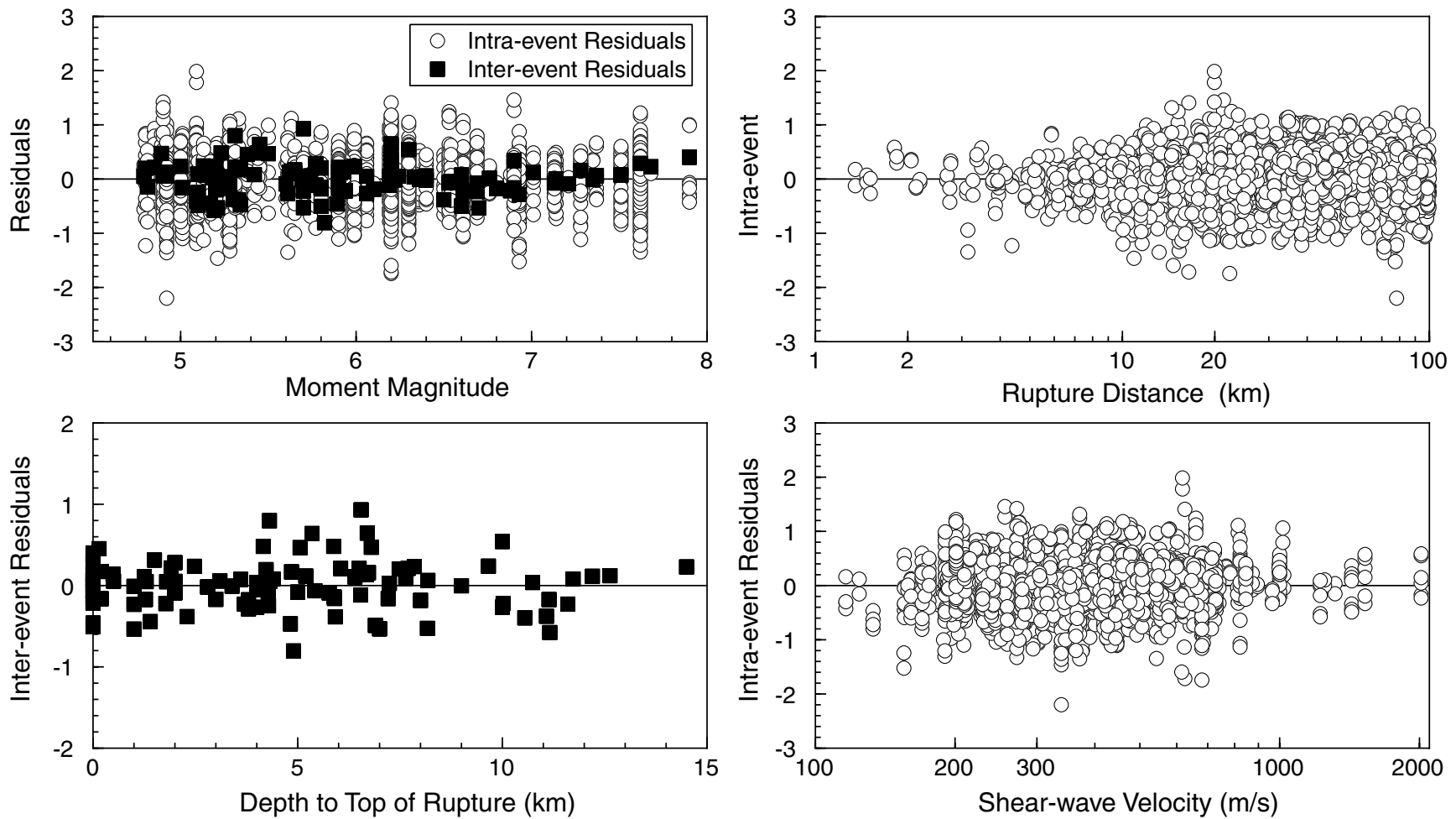

Figure 1. Residual plots for significant duration $D_{\mathrm{SR}}(5-75 \%)$. 

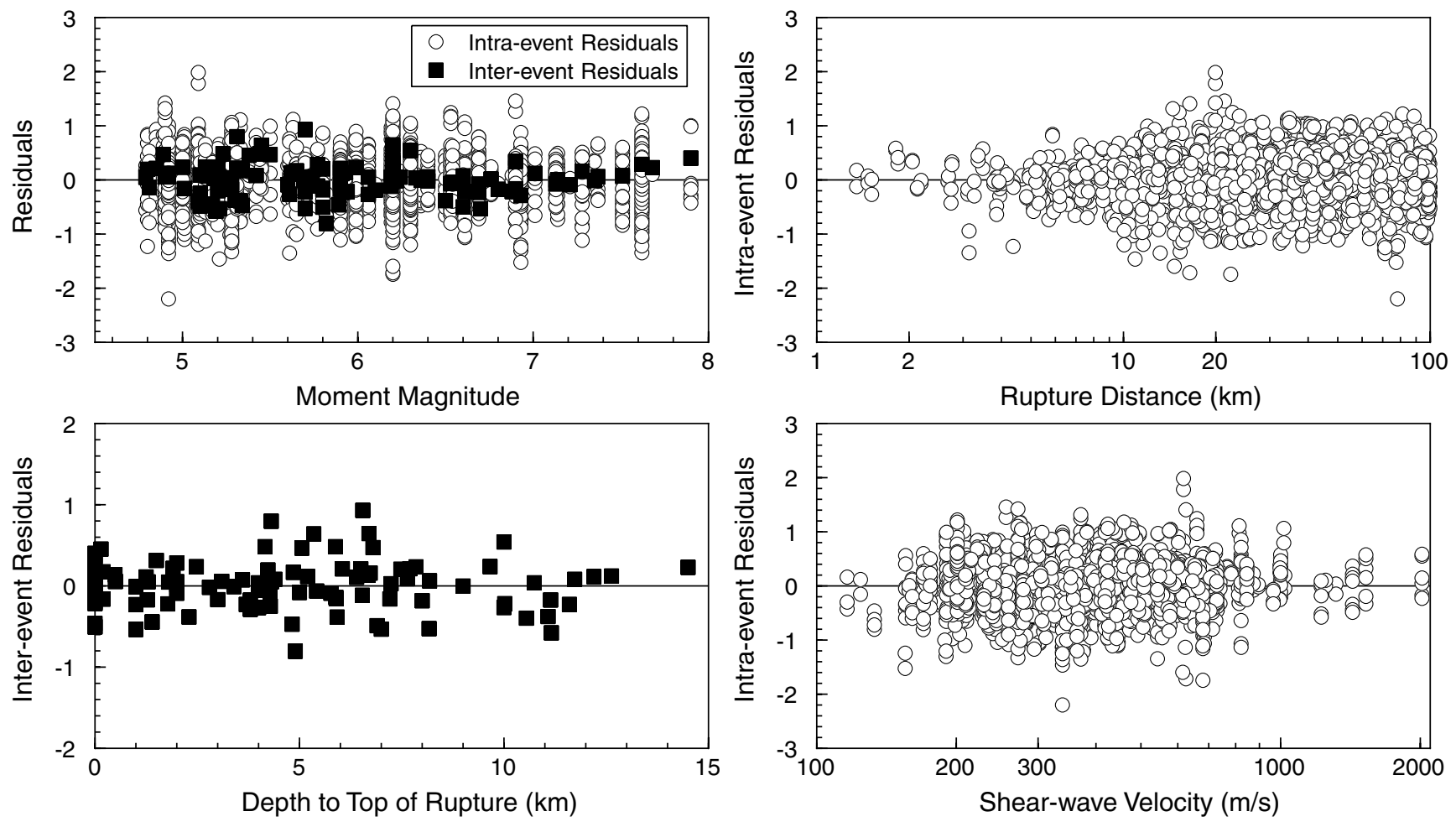

Figure 2. Residual plots for significant duration $D_{\mathrm{SR}}(5-95 \%)$.

will exceed the absolute threshold for the duration definition being considered. An empirical predictive model for PGA is thus required, and we have adopted the NGA model of Abrahamson and Silva (2008) for this purpose. The Abrahamson and Silva (2008) model is an obvious choice given that the dataset used in this study is very similar to that used by these authors. That said, a small adjustment must still be made as although the datasets are very similar, they are not the same, and we wish to ensure that our estimates of the probability of observing a zero-valued duration are not biased. Furthermore, the variability must be adjusted as the study of Abrahamson and Silva (2008) combines the horizontal components in a manner different from the approach taken in this study. The adjustment that is required is discussed in Stafford (2008).

The same rationale that was used to arrive at the functional form for the equations for significant duration was followed in order to select those for the bracketed and uniform durations. However, one additional factor that was considered is that the bracketed and uniform durations are inherently far less predictable than the significant durations. Given this reality, we placed greater emphasis on the selection of simple functional forms. We adopted the philosophy that while it may be possible to identify more complex expressions with statistically significant terms, the resulting reduction in variance is very minor, and we struggle to provide physical arguments to support the inclusion of these additional expressions.
The same functional form was adopted for both bracketed and uniform duration as both measures behave in a similar manner. The final regression coefficients were obtained using purpose-built scripts in MATLAB, but the preliminary testing of functional forms was carried out using the nlme package of $\mathrm{R}$. The final functional forms for bracketed and uniform duration are given in equations (8) and (9), respectively:

$$
\begin{aligned}
\ln D_{\mathrm{BA}}(x)= & c_{0}+m_{1} M_{\mathrm{w}}+r_{1} \ln \sqrt{R_{\mathrm{rup}}^{2}+h_{1}^{2}} \\
& +v_{1} \ln V_{S 30}+f_{1} F_{r v}, \\
\ln D_{\mathrm{UA}}(x)= & c_{0}+m_{1} M_{\mathrm{w}}+r_{1} \ln \sqrt{R_{\mathrm{rup}}^{2}+h_{1}^{2}} \\
& +v_{1} \ln V_{S 30}+f_{1} F_{r v} .
\end{aligned}
$$

Bommer et al. (2003) have discussed the various ways in which the style-of-faulting influences ground-motion amplitudes. Typically, a distinction is made between normal, strike-slip, and reverse-faulting events. However, in equations (8) and (9), a factor is only included to increase the bracketed and uniform durations associated with reversefaulting events. A distinction between strike-slip and normal events was considered but was found to be statistically insignificant. The increase in duration associated with reversefaulting events can easily be explained by virtue of the fact 
that ground-motion amplitudes increase for these types of events and that the threshold acceleration level is therefore likely to be exceeded for longer periods of time.

As previously mentioned, regression is performed using the individual components and the component-to-component standard deviation, $\sigma_{c}$, is estimated using all records for which both horizontal components are nonzero valued. The number of records for which this condition holds is given in Table 1. The final coefficients for the models defined by equations (8) and (9) are given in Tables 3 and 4, respectively. In these tables, in addition to the coefficients and variance components that were given in Table 2 for the significant durations, two correlation coefficients are also provided, $\rho_{\eta}$ and $\rho_{\varepsilon}$. The correlation coefficients are estimated directly as part of the regression approach of Stafford (2008). The $\rho_{\eta}$ value represents the correlation between the interevent residuals of the predictions of PGA and the duration measure in question, while the $\rho_{\varepsilon}$ value represents the corresponding correlation between the intraevent residuals.

The final consideration in the development of the models was to explore the inclusion of rupture directivity effects. Somerville et al. (1997) showed that forward rupture directivity generally produces motions of shorter duration as a result of the almost simultaneous arrival of waves emanating from different portions of the fault rupture, whereas in the backward directivity region the separation of these waves results in signals of extended duration. Somerville et al. (1997) derived factors for the adjustment of empirical predictions of $D_{\mathrm{SR}}(5-75 \%)$ based on the proportion of the total rupture between the epicenter and the recording station and the azimuth of the epicenter-station path with respect to the fault strike for strike-slip events; for dip-slip events an analogous parameterization was suggested. Within the framework of the NGA project, Spudich and Chiou (2008) developed new rupture directivity factors for the adjustment of predicted spectral ordinates from the NGA equations. The Spudich and Chiou
(2008) study aimed to address some shortcomings identified in the formulation of Somerville et al. (1997) and defined an isochrone directivity parameter (IDP). The intraevent residuals of the durations predicted by the equations developed in this study were examined for trends with respect to the IDP of Spudich and Chiou (2008). For the significant duration $D_{\mathrm{SR}}(5-75 \%)$, Figure 3 shows the residuals with respect to IDP for data grouped into magnitude and distance bins, which indicate that there are no clear and consistent trends with respect to this parameter, particularly in those bins (larger magnitudes and shorter distances) where rupture directivity effects would be expected to be most pronounced. Similar considerations were also made for the absolute durations, for which it was possible to observe some weak trends. However, the aleatory variability that exists for these measures of duration is very large, and the increase in complexity resulting from the introduction of terms for modeling directivity effects is not warranted. As the reduction in aleatory variability that results from the inclusion of the directivity terms is very minor, it was considered preferable to maintain as simple a functional form as possible. For this reason, rupture directivity has not been included as an explanatory variable in the models presented herein. It should be noted that Kempton and Stewart (2006) presented a model to account for rupture directivity effects. They initially attempted to incorporate the rupture directivity parameter (RDP) proposed by Somerville et al. (1997) but found no significant correlation with this parameter, except for the case of strike-slip ruptures and only for forward directivity (i.e., no corresponding increase in duration was found for the backward directivity region). Kempton and Stewart (2006) eventually proposed a linear adjustment (having a negative correlation with increasing distance out to $20 \mathrm{~km}$ from the source) to their basemodel predictions in order to account for forward directivity effects. However, Kempton and Stewart (2006) state that this adjustment may be interpreted as a directivity effect for

Table 3

Parameters of the Regression Models for Bracketed Durations with Absolute Thresholds of $0.025,0.050$, and $0.100 \mathrm{~g}$

\begin{tabular}{cccc}
\hline Parameter & $D_{\mathrm{BA}}(0.025 g)$ & $D_{\mathrm{BA}}(0.050 g)$ & $D_{\mathrm{BA}}(0.100 g)$ \\
\hline$c_{0}$ & $9.6688 \pm 3.463$ & $3.0982 \pm 1.6243$ & $0.6342 \pm 1.8931$ \\
$m_{1}$ & $1.3798 \pm 0.0546$ & $1.6885 \pm 0.0849$ & $1.7122 \pm 0.1315$ \\
$r_{1}$ & $-3.1204 \pm 0.7236$ & $-2.2715 \pm 0.3376$ & $-2.7126 \pm 0.369$ \\
$h_{1}$ & $46.3141 \pm 20.8243$ & $19.3897 \pm 7.3035$ & $11.1824 \pm 4.292$ \\
$v_{1}$ & $-0.6247 \pm 0.0895$ & $-0.7994 \pm 0.1339$ & $-0.5269 \pm 0.2161$ \\
$f_{1}$ & $0.173 \pm 0.0863$ & $0.145 \pm 0.1287$ & $0.1486 \pm 0.1941$ \\
$\tau$ & $0.5017 \pm 0.0076$ & $0.5652 \pm 0.0138$ & $1.0273 \pm 0.0669$ \\
$\sigma$ & $1.0265 \pm 0.0065$ & $1.2743 \pm 0.0113$ & $1.3983 \pm 0.0222$ \\
$\sigma_{c}$ & $0.4478 \pm 0.0146$ & $0.597 \pm 0.0232$ & $0.7261 \pm 0.0393$ \\
$\sigma_{T, \mathrm{ARB}}$ & $1.2271 \pm 0.0033$ & $1.5165 \pm 0.0058$ & $1.8809 \pm 0.0371$ \\
$\sigma_{T, \mathrm{GM}}$ & $1.1425 \pm 0.0057$ & $1.394 \pm 0.0099$ & $1.7351 \pm 0.0164$ \\
$\rho_{\eta}$ & $0.0119 \pm 0.1844$ & $0.2211 \pm 0.1754$ & $0.6417 \pm 0.1085$ \\
$\rho_{\varepsilon}$ & $0.429 \pm 0.0263$ & $0.5076 \pm 0.028$ & $0.5193 \pm 0.0371$ \\
\hline
\end{tabular}

The plus and minus values define the approximate $95 \%$ confidence interval for each parameter. 
Table 4

Parameters of the Regression Models for Uniform Durations with Absolute Thresholds of $0.025,0.050$, and $0.100 \mathrm{~g}$

\begin{tabular}{crcr}
\hline Parameter & \multicolumn{1}{c}{$D_{\mathrm{UA}}(0.025 g)$} & \multicolumn{1}{c}{$D_{\mathrm{UA}}(0.050 g)$} & \multicolumn{1}{c}{$D_{\mathrm{UA}}(0.100 g)$} \\
\hline$c_{0}$ & $5.5325 \pm 1.2505$ & $3.626 \pm 1.1044$ & $0.6011 \pm 1.4784$ \\
$m_{1}$ & $1.5598 \pm 0.0613$ & $1.5675 \pm 0.0811$ & $1.536 \pm 0.1139$ \\
$r_{1}$ & $-2.6156 \pm 0.2603$ & $-2.5499 \pm 0.2144$ & $-2.603 \pm 0.2542$ \\
$h_{1}$ & $22.5475 \pm 5.5147$ & $12.6151 \pm 3.1416$ & $7.7907 \pm 2.4482$ \\
$v_{1}$ & $-0.9392 \pm 0.0914$ & $-0.9929 \pm 0.123$ & $-0.7645 \pm 0.1937$ \\
$f_{1}$ & $0.2275 \pm 0.0872$ & $0.207 \pm 0.1174$ & $0.2902 \pm 0.1746$ \\
$\tau$ & $0.6287 \pm 0.0123$ & $0.6758 \pm 0.0177$ & $0.784 \pm 0.0335$ \\
$\sigma$ & $1.07 \pm 0.0035$ & $1.1911 \pm 0.0057$ & $1.2856 \pm 0.0103$ \\
$\sigma_{c}$ & $0.3294 \pm 0.0108$ & $0.4018 \pm 0.0156$ & $0.456 \pm 0.0248$ \\
$\sigma_{T, \mathrm{ARB}}$ & $1.284 \pm 0.0061$ & $1.4272 \pm 0.0086$ & $1.5733 \pm 0.0173$ \\
$\sigma_{T, \mathrm{GM}}$ & $1.241 \pm 0.0029$ & $1.3694 \pm 0.0046$ & $1.5058 \pm 0.0075$ \\
$\rho_{\eta}$ & $0.0555 \pm 0.1838$ & $0.2482 \pm 0.173$ & $0.0097 \pm 0.1844$ \\
$\rho_{\varepsilon}$ & $0.7449 \pm 0.0143$ & $0.796 \pm 0.0139$ & $0.8079 \pm 0.0177$ \\
\hline
\end{tabular}

The plus and minus values define the approximate $95 \%$ confidence interval for each parameter.

strike-slip events and the $D_{\mathrm{SR}}(5-75 \%)$ duration definition, but that in other cases these terms are effectively correcting for a bias in the distance scaling of the base model. Figure 4 presents residuals grouped as in Figure 3 plotted against the RDP parameter, and once again no consistent and statistically significant trends are found. It therefore seems that although one may anticipate that rupture directivity should be incorporated into predictive equations for duration measures, we are not currently able to adequately capture this phenomenon with empirical models.

A final point worthy of note is that in order to explore the sensitivity of the magnitude scaling in our models, we performed the regressions with various subsets of the data, firstly removing all of the recordings from 1999 Chi-Chi earthquakes. Additionally, we performed the regressions after removing poorly recorded events (defined, in different experiments, as producing less than 5, less than 10, and less than 15 records) from both the datasets, with and without Chi-Chi. The coefficients do, of course, change as a result but the changes were neither systematic nor significant in terms of the medians; the only difference being, as expected, a reduction in the interevent standard deviation when poorly recorded events are removed. The results presented in the article, and recommended for use, are those obtained from the full dataset.

\section{New Predictive Equations and Comparison with Previous Studies}

As noted previously, there are relatively few published equations for the prediction of strong-motion durations, and many of those that are available are sufficiently old to be considered obsolete. This limits the scope for making comparisons of the predictions from the new equations with those from existing models, not least because some of the older models use such different parameter definitions that several adjustments would be required to achieve compatibility be- fore making the comparisons. Nonetheless, a few graphical comparisons are made in order to illustrate and assess the performance of the new equations.

In Figure 5 the new predictions for significant durations are shown for various combinations of magnitude and distance and compared with those from Abrahamson and Silva (1996) and Kempton and Stewart (2006). The first observation that can be made is the great similarity between the predictions from the other two equations, although this is not very surprising considering that they use very similar functional forms. As noted earlier, this form consists of some physically based reasoning, that is, that the significant duration at short distances is equivalent to the source duration and that the relationship that the source duration is approximately equal to the reciprocal of the corner frequency holds. The authors of the other equations then adjust this slightly to allow for a magnitude-dependent stress drop and include additive terms for both distance and site class, which may not capture well the dependence on distance.

Although not identical, the predictions from our equations are broadly consistent with those from the other equations at short distances from the source, where the duration of shaking is expected to be controlled by the duration of the rupture. The most important difference between the previously published models and our own is the dependence of the significant duration on distance. In the previous models, with increasing distance one observes decreasing amplitudes and an increased wave-train length. If the distribution of energy within the signal remains roughly constant with increasing distance, then the significant duration should continue to increase indefinitely as suggested by these models. The scaling that we obtain suggests that, initially, the increasing length of the wave train is important and that the energy is partitioned from direct arrivals into the indirect arrivals. In this case the Arias intensity consists of significant contributions from both of these wave types. However, after some distance the relative contribution to the Arias intensity of 

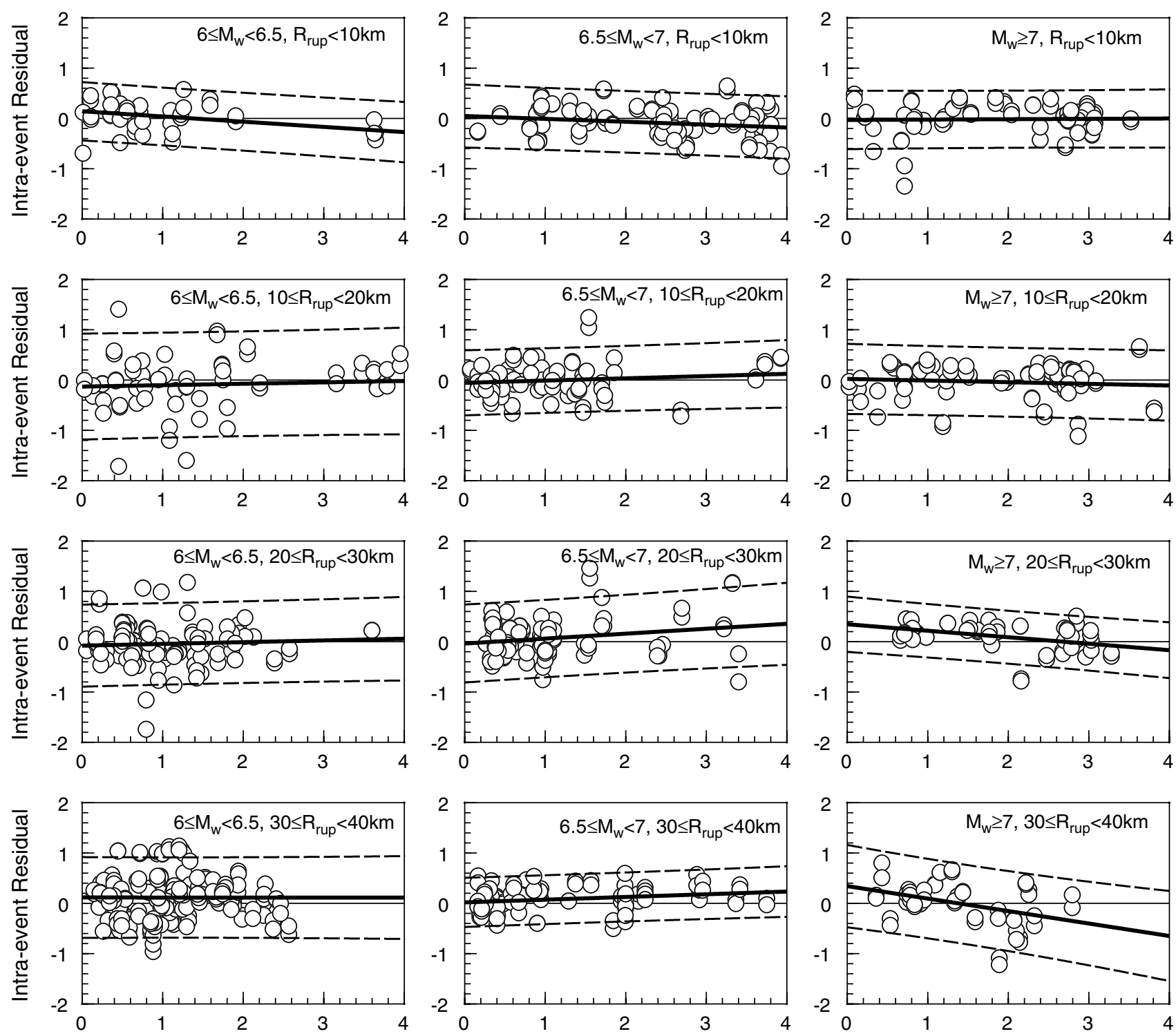

Isochrone Directivity Parameter (IDP)

Isochrone Directivity Parameter (IDP)

Isochrone Directivity Parameter (IDP)

Figure 3. Intraevent residuals for the $D_{\mathrm{SR}}(5-75 \%)$ significant duration model plotted against the IDP of Spudich and Chiou (2008). The residuals are grouped according to magnitude-distance bins as shown on the individual panels. The solid line represents a linear regression through the residuals, and the dashed lines represent the $95 \%$ prediction interval for this linear regression.

the indirect arrivals lessens. This may be due to much of these components being high-frequency waves whose amplitudes are decaying quite rapidly. The net effect is that the significant duration does not continue to increase indefinitely and that the bulk of the energy in the motion comes from wave arrivals occurring early in the signal. A degree of saturation of the duration with both magnitude and distance, as indicated by our models, seems reasonable.

A particular difference between our predictions and those from the earlier models is the absence of the marked increase that the previous models indicate as event size increases from magnitude 7 to 8 (where, it should be noted, the data are still relatively sparse) for distant sites. For very large earthquakes the length of the fault rupture becomes so large that sites close to one end of the fault are effectively unaware of the seismic energy radiated from the other end of the rupture. The empirical relationship of Wells and Coppersmith (1994) for strike-slip earthquakes indicates that for a magnitude 8 event the median fault rupture length would be $245 \mathrm{~km}$.

The crossover for the durations at different distances in the left-hand frames is obviously unexpected, but it is very small. The important point is that referring back to Figures 1 and 2, the residuals do not show any trend with magnitude or distance, suggesting that the model is appropriate. Although not shown, when we conduct a regression analysis using the 

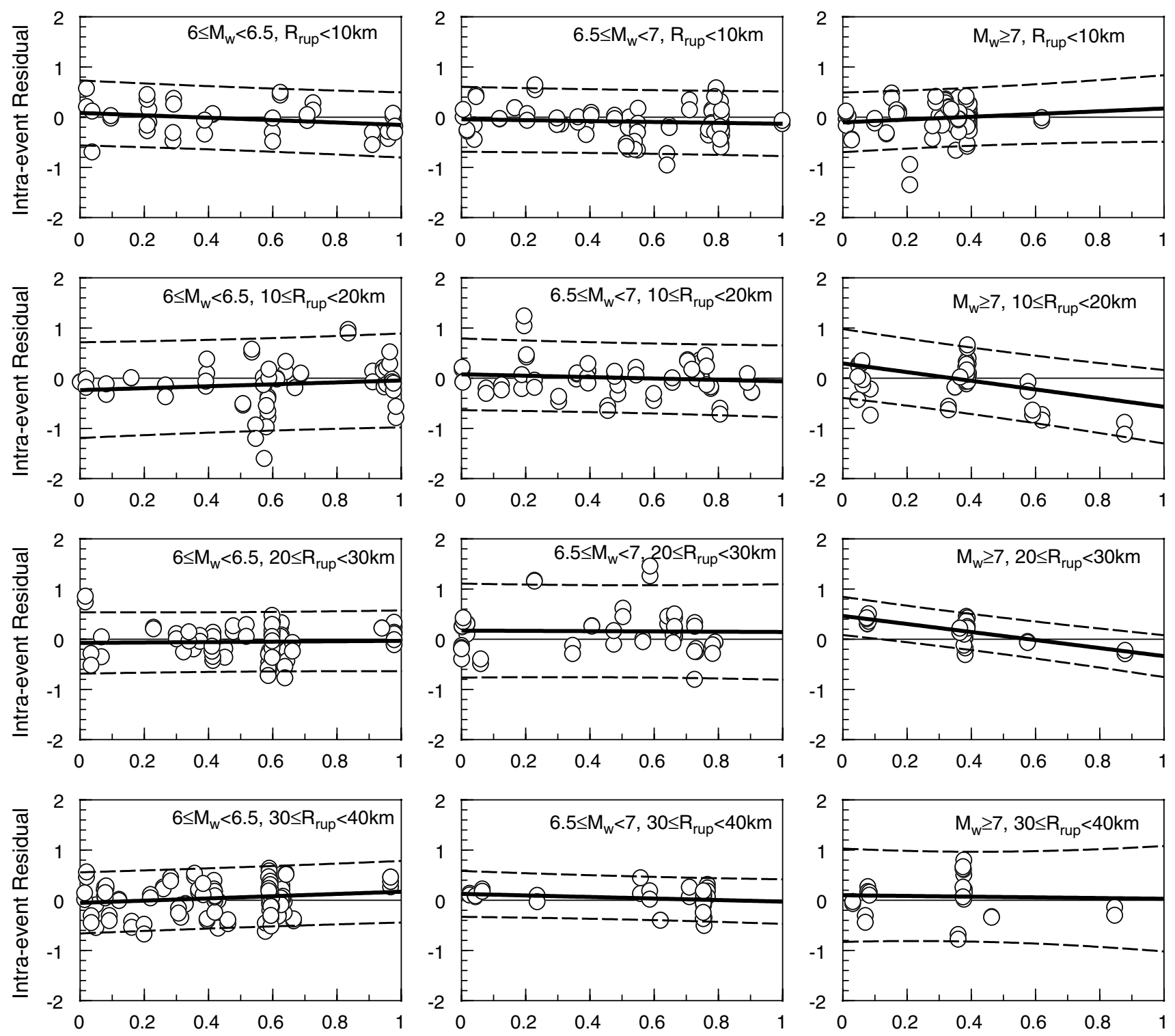

Rupture Directivity Parameter (RDP)

Rupture Directivity Parameter (RDP)

Rupture Directivity Parameter (RDP)

Figure 4. Intraevent residuals for the $D_{\mathrm{SR}}(5-75 \%)$ significant duration model plotted against the RDP of Somerville et al. (1997). The residuals are grouped according to magnitude-distance bins as shown on the individual panels. The solid line represents a linear regression through the residuals, and the dashed lines represent the $95 \%$ prediction interval for this linear regression.

functional form of Kempton and Stewart (2006) and the dataset used in this study, we find significant trends in the residuals with respect to both distance and shear-wave velocity. These findings provide us with confidence in departing from the functional form suggested by Abrahamson and Silva (1996) and adapted by Kempton and Stewart (2006).

By way of exploring the validity of the inferred magnitude and distance dependence in our models, the expected values of significant duration for different combinations of predictor variables are estimated from the model of Stafford et al. (2009) for an energy-based envelope function for earthquake accelerograms. Because this envelope function is di- rectly related to the Arias intensity, it can be used to calculate the significant durations based on build-up of this parameter. Figure 6 compares predicted median durations from the models presented in this article and from the envelope function of Stafford et al. (2009), from which it can be seen that the overall behavior, particularly in terms of saturation with magnitude and distance, is common to both approaches. Although this cannot be considered as independent validation of the new models, we believe this does provide corroboration for the models presented in this article.

The models for significant duration that are presented herein scale in a very simple way with respect to both 

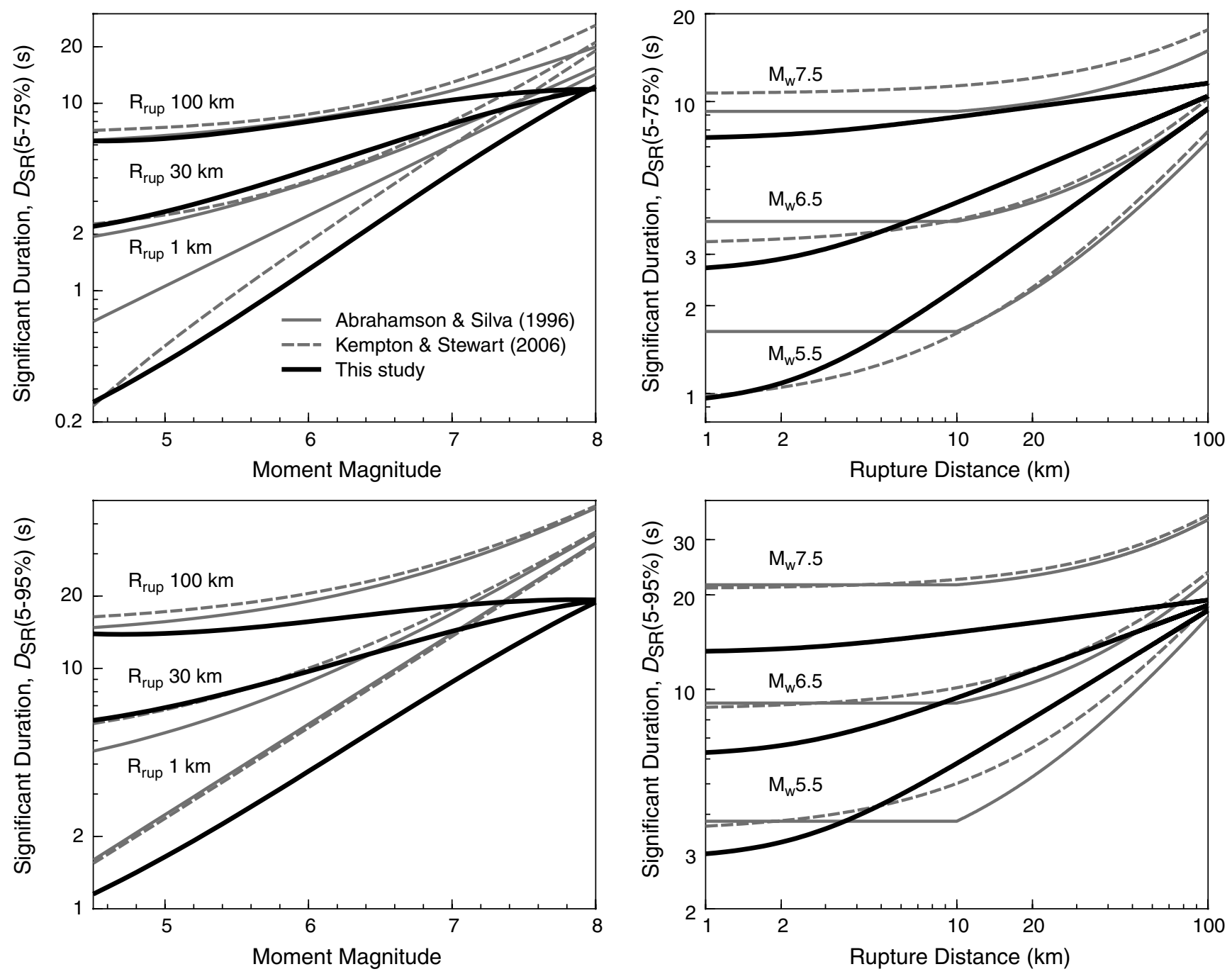

Figure 5. Comparison of empirical predictive models for significant duration. Here the comparison is made between the models of Abrahamson and Silva (1996), Kempton and Stewart (2006), and that of this study. For the plots against rupture distance, a depth to the top of a rupture of zero is assumed for the model of this study, and for the plots against magnitude this parameter is prescribed so that it decreases with increasing magnitude. The variation of $Z_{\mathrm{tor}}$ with $M_{\mathrm{w}}$ is modeled using a cubic polynomial fit through the data used to derive the regression model.

shear-wave velocity and the depth to the top of the rupture. Given that the $D_{\mathrm{SR}}(5-95 \%)$ is defined so as to capture the duration of the entire wave train, one might anticipate that the influence of local soil conditions would be more marked for this measure. However, Figure 7 presents plots of the significant durations with respect to both depth to the top of rupture and shear-wave velocity, and from inspection of this figure it may be appreciated that the scaling for both $D_{\mathrm{SR}}(5-75 \%)$ and $D_{\mathrm{SR}}(5-95 \%)$ is very similar in both cases.

With regard to the right-hand panels of Figure 7, it can be seen that the new models predicted a clear dependence on the shear-wave velocity with, as would be expected, longer durations on softer sites. Direct comparison with the $V_{S 30}$ dependence in the Kempton and Stewart (2006) model is difficult because whereas in our equations the influence of this parameter on duration is multiplicative, in their model it is additive. For the two bounding values of $V_{S 30}$ shown in Figure 7 (180 and $760 \mathrm{~m} / \mathrm{sec}$ ), Kempton and Stewart (2006) predict increases of 0.75 and $2.38 \mathrm{sec}$ for the $5-75 \%$ and $5-95 \%$ significant durations, respectively, which are on the same order as those from our model for most magnitude and distance ranges of engineering interest.

Figure 8 compares median predictions of the six durations based on absolute thresholds for which new equations have been derived, for three magnitudes and a range of distances. The behavior is as would be expected: the bracketed durations are consistently greater than the uniform durations for a given threshold. For a given accelerogram, both definitions yield a value of zero when the threshold is equal to the PGA and become equal to the total duration of the record as the threshold tends to zero, but for 

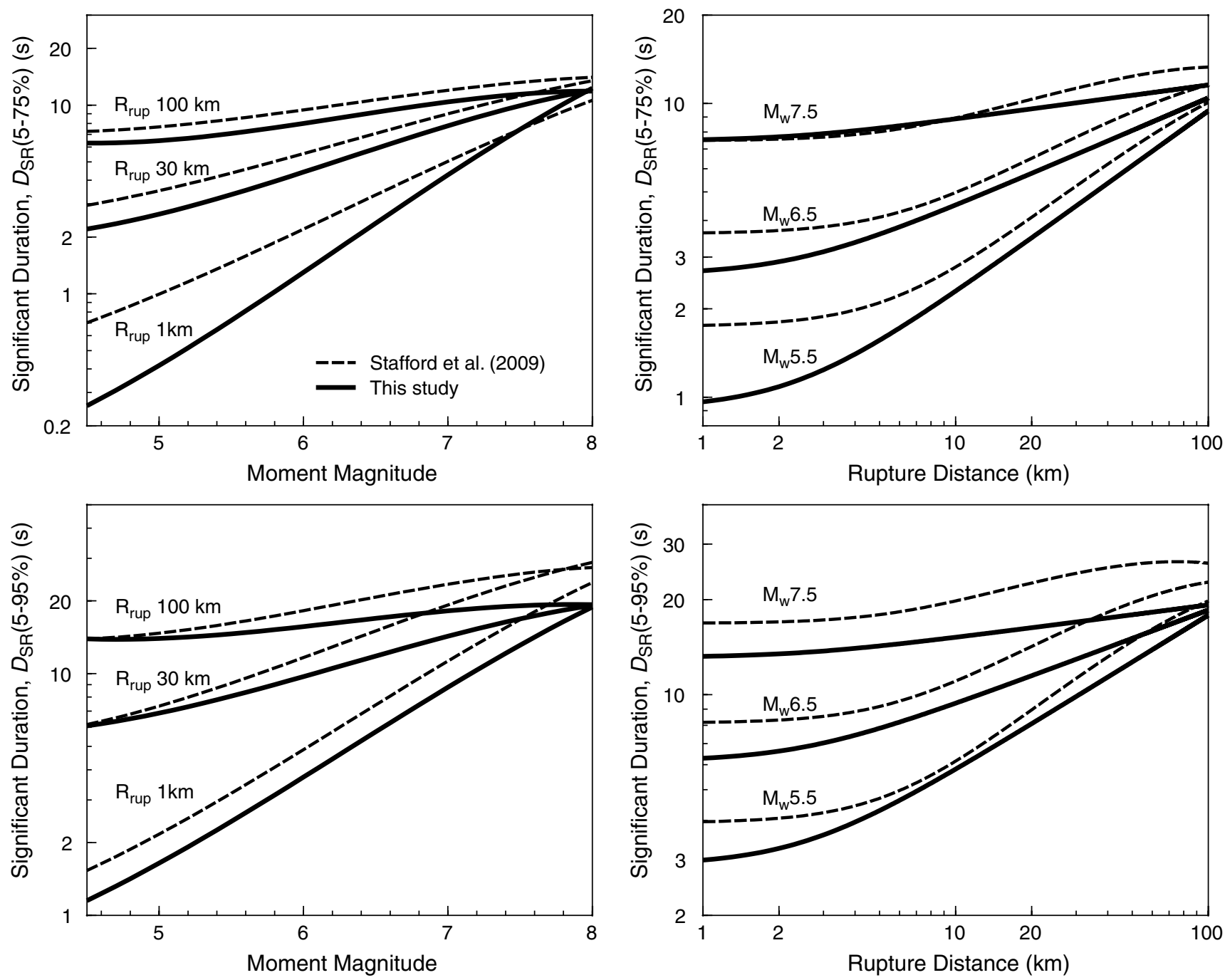

Figure 6. Comparison between the predictive models derived in this study with that inferred through the application of the energy-based envelope function of Stafford et al. (2009). The specification of the depth to the top of rupture is the same as in Figure 5.

all intermediate values $D_{\mathrm{BA}}$ is always larger than $D_{\mathrm{UA}}$, and this is confirmed by the predictions. Another expected feature of the predictions, following the previous discussion, is that at greater distances from the source, where the PGA level will be expected to reduce to close to the threshold accelerations, both durations should converge to zero. Although not immediately apparent in these plots on logarithmic axes, the differences between the $D_{\mathrm{BA}}(0.10 g)$ and $D_{\mathrm{UA}}(0.10 \mathrm{~g})$ durations at $100 \mathrm{~km}$ is a small fraction of a second for all magnitudes.

Figure 9 compares predictions of bracketed duration from the new equations with three previously published models, one derived from Japanese data (Kawashima and Aizawa, 1989) and two derived from Greek strong-motion data (Papazachos et al., 1992; Koutrakis et al., 2002). These comparisons are hampered by the use of different parameter definitions in the models; adjustments are made for magnitude scales as explained in the figure caption. There is a very high degree of divergence amongst the predictions, with those from the new models generally being appreciably lower than the durations obtained from the other models. Although we do not have any suggestions for why this might be the case, some of the durations predicted by the other models do seem highly suspect: for an earthquake of $M_{\mathrm{w}} 5.5$, the Papazachos et al. (1992) model predicts $20 \mathrm{sec}$ of shaking exceeding $0.1 \mathrm{~g}$ close to the source, and this value becomes greater than $100 \mathrm{sec}$ for an event of $M_{\mathrm{w}}$ 7.5. Another feature of the new models that differs from those published previously is the more rapid decay of the bracketed durations with distance, but this can be easily explained as a consequence of the present study implicitly accounting for the components of motion that yield durations of zero. All of the other studies have simply neglected these records and thus have positively biased (overestimated) predictions at increasing distances. The extent of this overprediction has been demonstrated by Stafford (2008). 

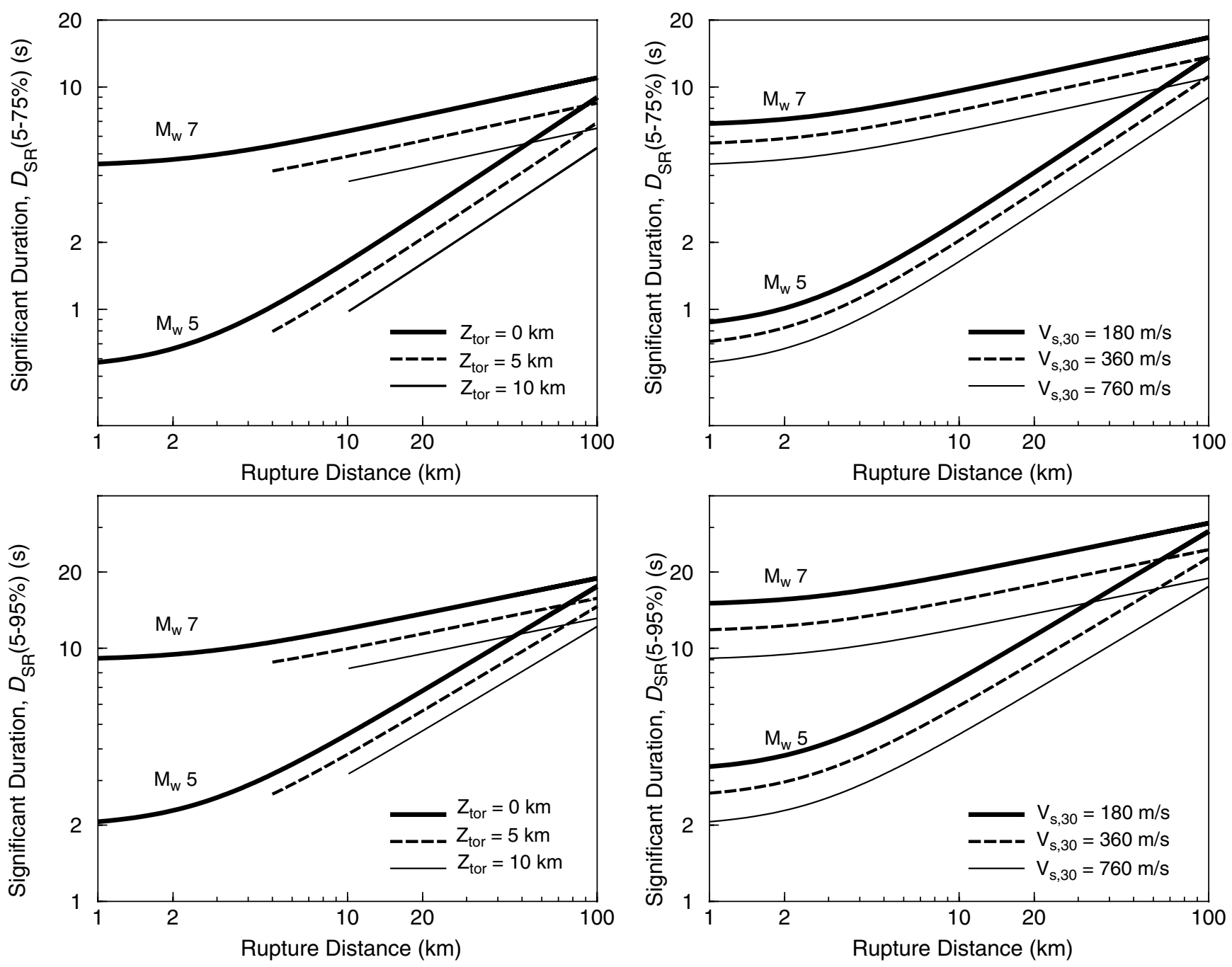

Figure 7. Scaling of the significant duration models with respect to the depth to the top of the rupture (left-hand panels) and shear-wave velocity (right-hand panels) for both $D_{\mathrm{SR}}(5-75 \%)$ and $D_{\mathrm{SR}}(5-95 \%)$. The values of shear-wave velocity have been selected to coincide with the boundaries of the NEHRP site classification scheme.

\section{Concluding Remarks}

This article has presented new equations for the prediction of eight different measures of ground-motion duration, applicable to sites at up to $100 \mathrm{~km}$ from shallow crustal earthquakes of magnitude from $M_{\mathrm{w}} 4.8$ to 7.9. The models are based on simple functional forms and include only a small number of explanatory variables, achieving what we consider a good balance between reduced aleatory variability and model complexity. The models include magnitude, distance, site classification $\left(V_{S 30}\right)$, and either depth-to-top-of-rupture or style-of-faulting depending on the duration definition under consideration. Interestingly, the influence of fault rupture directivity does not appear strong enough to warrant its inclusion in the models, because only a very modest reduction in variability is achieved for a large increase of complexity. For situations in which it is thought that rupture directivity effects may significantly alter the predicted duration values, numerical analyses may be required as current empirical models are not able to capture this effect in a robust manner.

The new models for duration measures based on absolute acceleration thresholds appear to perform consistently better than previously published models for similar measures of strong-motion duration. The new models for significant durations predict values that are broadly comparable to those obtained from other relatively recent models but do not predict durations that increase monotonically with increasing distance. For the case of bracketed and absolute durations, we are of the view that the new models should always be used in preference to the previously published equations, which we believe are generally flawed, and this is an important contribution of this article to have provided robust models for these parameters. For the case of significant durations, however, we do not believe that our models necessarily supersede those of Kempton and Stewart (2006), but rather represent an alternative interpretation of the same dataset. 

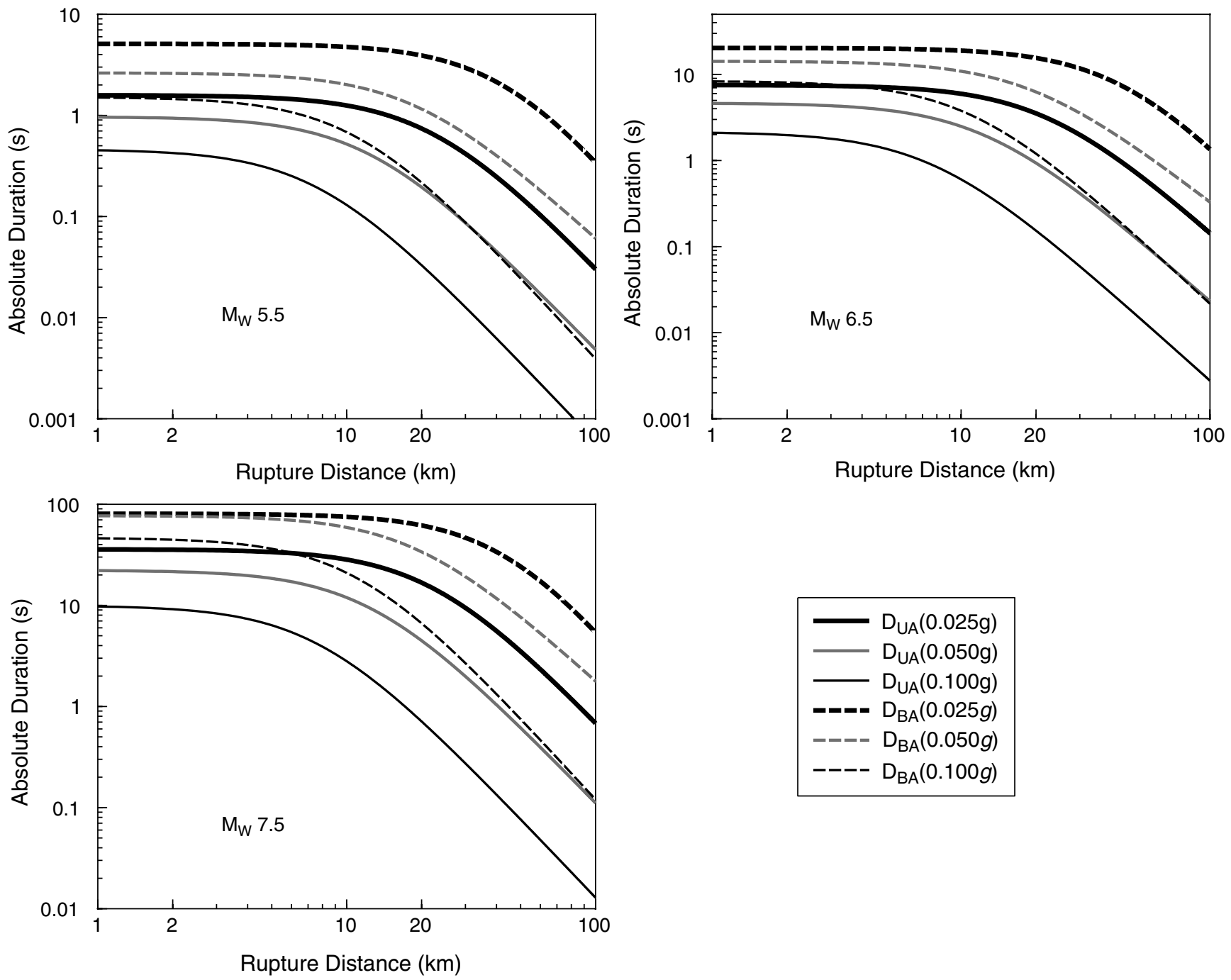

Figure 8. Comparison of the general scaling with respect to magnitude and distance of the six different models for absolute duration developed in this study. The legend is common to all three panels. The predictions are made for a shear-wave velocity of $350 \mathrm{~m} / \mathrm{sec}$ and for strike-slip or normal rupturing events.

In that sense, the difference between our predictions could be considered to represent epistemic uncertainty in the estimation of this parameter. Therefore, a legitimate approach for seismic hazard assessment in terms of significant duration in regions of shallow crustal seismicity would be to combine these equations within a logic-tree framework in the same way as would be done for spectral accelerations (Bommer et al., 2005).

We believe that these new equations can be applied with confidence in active regions of shallow crustal seismicity, within the ranges of applicability in terms of magnitude and distance (Bommer et al., 2007). The equations have been derived using the NGA dataset, which is dominated by recordings from western North America and from Taiwan, but also includes data from other active crustal regions including southern Europe, Turkey, and the Middle East. The NGA database was compiled for the specific purpose of deriving spectral acceleration prediction equations primar- ily for application in coastal California, but it has been shown that they are also applicable to the prediction of response spectrum ordinates in Europe and the Middle East (Stafford, Strasser, and Bommer, 2008). Although no specific tests using data from the latter region have been carried out, there is no obvious reason why the equations presented in this article should not be used for the estimation of duration of shaking from shallow crustal earthquakes in Europe and the Middle East, and indeed any other active crustal region.

Because an estimation of duration would usually be required in conjunction with other parameters of the ground motion, probably related to amplitude, caution must be applied in how durations are calculated, especially within the framework of probabilistic seismic hazard analysis (PSHA). If PSHA is used to determine ground motions for design or assessment, then rather than employing these equations directly in the hazard calculations, the hazard in terms 

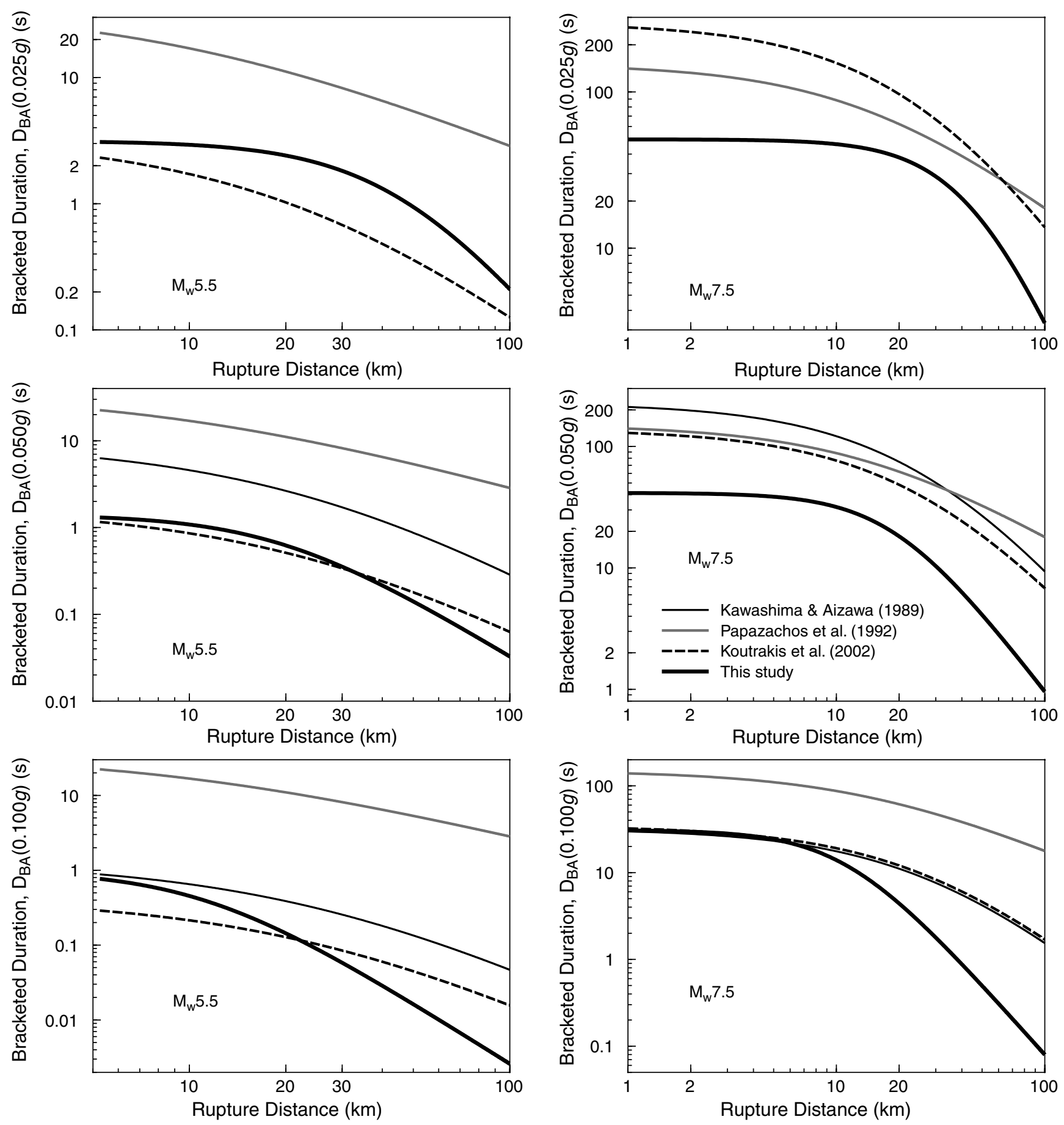

Figure 9. Comparison of empirical predictive models for bracketed duration with different absolute acceleration thresholds. Predictions are made for a site located perpendicular to a strike-slip fault rupture. For the $M_{\mathrm{w}} 5.5$ case the depth to the top of rupture is $5.3 \mathrm{~km}$, while for the $M_{\mathrm{w}} 7.5$ case the depth is $0.2 \mathrm{~km}$. The model of Kawashima and Aizawa (1989) has magnitudes converted from $M_{\mathrm{JMA}}$ to $M_{\mathrm{w}}$ using the relationship of Fukushima (1996), while the models of Papazachos et al. (1992) and Koutrakis et al. (2002) have $M_{S}$ values converted to $M_{\mathrm{w}}$ using the conversion of Ambraseys and Free (1997).

of the amplitude-based parameter (such as the spectral ordinate at the fundamental period of the structure of interest) should be disaggregated in order to determine the dominant earthquake scenario in terms of magnitude and distance from the site. The equations presented in this article can then be employed to calculate the duration corresponding to this scenario. A preferable approach would be to calculate the hazard in terms of a vector of the amplitude-based parameter and the duration simultaneously. This requires coupled predictions of duration and other strong-motion parameters including 
the correlations of their residuals. The next stage of this work will develop such models.

\section{Data and Resources}

The accelerograms and the associated metadata that were used in this study were obtained from the Pacific Earthquake Engineering Research (PEER) NGA strong-motion database at http://peer.berkeley.edu/nga/ (last accessed July 2009).

\section{Acknowledgments}

We are very grateful to associate editor Arben Pitarka, Jonathan Stewart, and an anonymous reviewer for insightful and constructive feedback on the first version of this manuscript that led to very appreciable improvement of the article. The second author is a Fellow of the Willis Research Network and that financial support is noted with gratitude. The third author was partially funded by the $\mathrm{Al} \beta \mathrm{an}$ program of the European Union and this assistance is also gratefully acknowledged.

\section{References}

Abrahamson, N. A., and W. J. Silva (1996). Empirical ground motion models, Report to Brookhaven National Laboratory.

Abrahamson, N., and W. Silva (2008). Summary of the Abrahamson and Silva NGA ground-motion relations, Earthq. Spectra 24, no. 1, 67-97.

Ambraseys, N. N., and M. W. Free (1997). Surface-wave magnitude calibration for European region earthquakes, J. Earthq. Eng. 1, no. $1,1-22$.

Baker, J. W., and C. A. Cornell (2006). Which spectral acceleration are you using?, Earthq. Spectra 22, no. 2, 293-312.

Bazzurro, P., and C. A. Cornell (2002). Vector-valued probabilistic seismic hazard analysis (VPSHA), Proc. of the 7th U.S. National Conference on Earthquake Engineering, Boston, Massachusetts, 21-25 July 2002.

Beyer, K., and J. J. Bommer (2006). Relationships between median values and aleatory variabilities for different definitions of the horizontal component of motion, Bull. Seismol. Soc. Am. 94, no. 4A, 1512-1522 (Erratum 2007, 97, no. 5, 1769)

Bommer, J. J., and A. Martínez-Pereira (1999). The effective duration of earthquake strong motion, J. Earthq. Eng. 3, no. 2, 127-172.

Bommer, J. J., and R. Mendis (2005). Scaling of displacement spectral ordinates with damping ratios, Earthq. Eng. Struct. Dyn. 34, no. 2, $145-165$.

Bommer, J. J., J. Douglas, and F. O. Strasser (2003). Style-of-faulting in ground-motion prediction equations, Bull. Earthq. Eng. 1, no. 2, 171-203.

Bommer, J. J., J. Hancock, and J. E. Alarcón (2006). Correlations between duration and number of effective cycles of earthquake ground motion, Soil Dyn. Earthq. Eng. 26, no. 1, 1-13.

Bommer, J. J., G. Magenes, J. Hancock, and P. Penazzo (2004). The influence of strong-motion duration on the seismic response of masonry structures, Bull. Earthq. Eng. 2, no. 1, 1-26.

Bommer, J. J., F. Scherbaum, H. Bungum, F. Cotton, F. Sabetta, and N. A. Abrahamson (2005). On the use of logic trees for ground-motion prediction equations in seismic hazard assessment, Bull. Seismol. Soc. Am. 95, no. 2, 377-389.

Bommer, J. J., P. J. Stafford, J. E. Alarcón, and S. Akkar (2007). The influence of magnitude range on empirical ground-motion prediction, Bull. Seismol. Soc. Am. 97, no. 6, 2152-2170.

Boore, D. M. (2005). Erratum: Equations for estimating horizontal response spectra and peak accelerations from western North American earthquakes: A summary of recent work, Seism. Res. Lett. 76, no. 3, 368-369.
Boore, D. M., J. Watson-Lamprey, and N. A. Abrahamson (2006). Orientation-independent measures of ground motion, Bull. Seismol. Soc. Am. 96, no. 4, 1502-1511.

BSSC (2004). NEHRP provision for seismic regulations for new buildings and other structures, 2003 edition, Report FEMA 450, Building Seismic Safety Council, Washington D.C.

Chiou, B., R. Darragh, N. Gregor, and W. Silva (2008). NGA project strong-motion database, Earthq. Spectra 24, no. 1, 23-44.

Douglas, J. (2003). Earthquake ground motion estimation using strongmotion records: A review of equations for the estimation of peak ground accelerations and response spectral ordinates, Earth-Science Rev. 61, 43-104.

Fukushima, Y. (1996). Scaling relations for strong ground motion prediction models, Bull. Seismol. Soc. Am. 86, no. 2, 329-336.

Green, R. A., and G. A. Terri (2005). Number of equivalent cycles concept for liquefaction evaluations-revisited, J. Geotech. Geoenviron. Eng. 131, no. 4, 477-488.

Hancock, J., and J. J. Bommer (2005). The effective number of cycles of earthquake ground motion, Earthq. Eng. Struct. Dyn. 34, no. 6, 637-664.

Hancock, J., and J. J. Bommer (2006). A state-of-knowledge review of the influence of strong-motion duration on structural damage, Earthq. Spectra 22, no. 3, 827-845.

Hancock, J., and J. J. Bommer (2007). Using spectral matched records to explore the influence of strong-motion duration on inelastic structural response, Soil Dyn. Earthq. Eng. 27, no. 4, 291-299.

Kagawa, T., K. Irikura, and P. G. Somerville (2004). Differences in ground motion and fault rupture process between the surface and buried rupture earthquakes, Earth Planets Space 56, 3-14.

Kawashima, K., and K. Aizawa (1989). Bracketed and normalized durations of earthquake ground accelerations, Earthq. Eng. Struct. Dyn. 19, 1041-1051.

Kempton, J. J., and J. P. Stewart (2006). Prediction equations for significant duration of earthquake ground motions considering site and nearsource effects, Earthq. Spectra 22, no. 4, 985-1013.

Koutrakis, S. I., G. F. Karakaisis, P. M. Hatzidimitriou, P. K. Kolipoulos, and V. N. Margaris (2002). Seismic hazard in Greece based on different strong ground motion parameters, J. Earthq. Eng. 6, no. $1,75-109$.

Liu, A., J. P. Stewart, N. Abrahamson, and Y. Morikawi (2001). Equivalent number of uniform stress cycles for liquefaction analysis, J. Geotech. Geoenviron. Eng. 127, no. 12, 1017-1026.

Papazachos, B. C., Ch. A. Papaiannou, V. N. Margaris, and N. P. Theodulidis (1992). Seismic hazard assessment in Greece based on strong motion duration, Proc. of 10th World Conference on Earthquake Engineering, Madrid, vol. 2, 425-430.

Pinheiro, J., D. Bates, S. DebRoy, D. Sarkar, and the R Core team (2008). nlme: Linear and nonlinear mixed effects models, $\mathrm{R}$ package version 3.1-89.

Priestley, M. J. N., G. M. Calvi, and M. J. Kowalsky (2007). Displacement-Based Seismic Design of Structures IUSS Press, Pavia, Italy, $721 \mathrm{pp}$.

R Core Development Team (2008). R: A language and environment for statistical computing, R Foundation for Statistical Computing, Vienna, Austria, ISBN 3-900051-07-0, http://www.R-project.org.

Somerville, P. G., N. F. Smith, R. W. Graves, and N. A. Abrahamson (1997). Modification of empirical strong ground motion attenuation relations to include the amplitude and duration effects of rupture directivity, Seism. Res. Lett. 68, no. 1, 199-222.

Spudich, P., and B. S. J. Chiou (2008). Directivity in NGA earthquake ground motions: Analysis using isochrone theory, Earthq. Spectra 24, no. 1, 279-298.

Stafford, P. J. (2008). Conditional prediction of absolute durations, Bull. Seismol. Soc. Am. 98, no. 3, 1588-1594.

Stafford, P. J., and J. J. Bommer (2009). Empirical equations for the prediction of the equivalent number of cycles of earthquake ground motion, Soil Dyn. Earthq. Eng. 29, no. 11/12, 1425-1436. 
Stafford, P. J., R. Mendis, and J. J. Bommer (2008). Dependence of damping correction factors for response spectra on duration and numbers of cycles, J. Struct. Eng.-ASCE 134, no. 8, 1364-1373.

Stafford, P. J., S. Sgobba, and G. C. Marano (2009). An energy-based envelope function for the stochastic simulation of earthquake accelerograms, Soil Dyn. Earthq. Eng. 29, no. 7, 1123-1133.

Stafford, P. J., F. O. Strasser, and J. J. Bommer (2008). An evaluation of the applicability of the NGA models to ground-motion prediction in the Euro-Mediterranean region, Bull. Earthq. Eng. 6, no. 2, 149-177.

Watson-Lamprey, J. A., and D. M. Boore (2007). Beyond $S a_{\text {GMRotI }}$ : Conversion to $S a_{\mathrm{Arb}}, S a_{\mathrm{SN}}$, and $S a_{\mathrm{MaxRot}}$, Bull. Seismol. Soc. Am. 97, no. 5, $1511-1524$.

Wells, D. L., and K. J. Coppersmith (1994). New empirical relationships among magnitude, rupture length, rupture width, rupture area, and surface displacement, Bull. Seismol. Soc. Am. 84, 974-1002.
Department of Civil and Environmental Engineering Imperial College London

South Kensington Campus

London SW7 2AZ, UK

p.stafford@imperial.ac.uk

$$
\text { (J.J.B., P.J.S.) }
$$

AIR Worldwide

New London House, 6 London Street

London EC3R 7LP, UK

(J.E.A.)

Manuscript received 3 October 2008 